\title{
Non-invasive investigation of Spironucleus vortens transmission in freshwater angelfish Pterophyllum scalare
}

\author{
C. F. Williams*, A. R. Vacca, D. Lloyd, B. Schelkle, J. Cable \\ School of Biosciences, Cardiff University, Main Building, Museum Avenue, Cardiff CF10 3AT, UK
}

\begin{abstract}
Spironucleus vortens is a protozoan fish parasite of veterinary and economic importance in the ornamental aquaculture industry. Despite this, key aspects of the life cycle of this organism, including its mode of transmission, have not been fully elucidated. We developed a non-invasive method for quantifying $S$. vortens in freshwater angelfish, which was then used to investigate parasite transmission and aggregation within host populations. As previously observed for $S$. meleagridis and $S$. salmonis, motile $S$. vortens trophozoites were detected in host faeces using light microscopy. Species-level identification of these flagellates was confirmed using 16S rDNA PCR. Faecal trophozoite counts were significantly correlated with trophozoite counts from the posterior intestine, the primary habitat of the parasite. This novel finding allowed effective prediction of intestinal parasite load from faecal counts. Overall, faecal count data revealed that $20 \%$ of hosts harbour $83 \%$ of parasites, conforming to the Pareto Principle (80/20 rule) of parasite aggregation with implications for parasite transmission. Trophozoites survived for $\geq 36 \mathrm{~d}$ outside the host within faeces and remained motile at low $\mathrm{pH}$ (comparable with that of angelfish stomach). No putative $S$. vortens cysts were observed in cultures or faecal samples. This calls into question the commonly accepted hypothesis that a protective cyst is required in the life cycle of $S$. vortens to facilitate transmission to a new host.
\end{abstract}

KEY WORDS: Diplomonad · Spironucleosis · Protozoan · Parasite $\cdot$ Trophozoite $\cdot$ Cyst $\cdot$ Aquaculture

\section{INTRODUCTION}

Ornamental aquaculture production is a global multi-billion dollar industry. International export trades in this sector have increased at an average growth rate of $14 \%$ per annum since 1985 (FAO 2005), with world exports of fish and fishery products (food fish included) reaching US\$102 billion in 2008 (FAO 2010). Disease is a major limiting factor in aquaculture production, causing losses of ca. $45 \%$ to the industry (FDA 2012). However, accurate diagnosis of many fish diseases requires sophisticated equipment, such as electron microscopy, and consultation with fish health experts for pathogen identification (Sterud \& Poynton 2002, Noga 2010). A further complication is the lack of available data on basic aspects of pathogen biology, including the life cycle and transmission of some disease-causing agents. Hence, infections are often undiagnosed, and may lead to disease outbreaks and significant mortality of fish if control measures fail.

Diplomonads are a group of aerotolerant anaerobic or microaerophilic organisms which inhabit low- $\mathrm{O}_{2}$ environments such as lake sediments and the intestinal tracts of animals (Biagini et al. 1998, Lloyd et al. 2002, Kolisko et al. 2010, Williams et al. 2011). Five species of piscine Spironucleus spp. are currently recognised: $S$. salmonicida, $S$. barkhanus and $S$. torosa infect marine food fish, whereas $S$. salmonis and $S$. vortens, the latter being the focus of the cur- 
rent study, infect freshwater ornamental fish (reviewed by Williams et al. 2011). First isolated from an angelfish in Florida (USA) in 1991 (Poynton et al. 1995), $S$. vortens is particularly prevalent in cichlids and cyprinids, including angelfish Pterophyllum scalare and discus Symphysodon discus (see Williams et al. 2011). The primary habitat of this parasite is the middle to posterior intestinal tract; however, motile trophozoites have also been isolated from other organs, including the liver, spleen and kidneys, indicating systemic spironucleosis. Recovery of this organism from head lesions has led to this parasite being described as the putative causative agent of hole-inthe-head disease (Paull \& Matthews 2001).

Accurate identification of Spironucleus spp. has been greatly hampered by lack of appropriate experimental methods. Earlier studies, which relied on the use of light microscopy alone to identify diplomonads, resulted in the assignation of piscine diplomonads to the genera Hexamita, Octomitus and Spironucleus. Recent detailed ultrastructural work and molecular tools, however, have confirmed that all piscine diplomonads belong to the genus Spironucleus (see Poynton \& Sterud 2002, Jørgensen \& Sterud 2007). PCR and/or sequencing primers have now been designed for most Spironucleus spp. (reviewed by Williams et al. 2011). In the case of $S$. vortens, however, the only available primers indiscriminately amplify the $16 \mathrm{~S}$ rRNA gene of most piscine Spironucleus species. Species-level identification of $S$. vortens therefore requires sequencing of $16 \mathrm{~S}$ rDNA and transmission electron microscopy.

The life cycle of piscine Spironucleus spp. is poorly characterised, with most information being inferred from the distantly related mammalian diplomonad parasite Giardia (reviewed by Ankarklev et al. 2010). Like Giardia, the life cycle of Spironucleus spp. is thought to be direct, via the faecal-oral route, with transmission between hosts being facilitated by an infective cyst (Woo \& Poynton 1995). The cyst form has been well-documented for $S$. muris and $S$. meleagridis, which infect rodents and birds, respectively (Januschka et al. 1988, Wood \& Smith 2005). Cysts of the piscine diplomonad $S$. salmonis (host: salmon, trout) have also been documented in vitro and in vivo (reviewed by Williams et al. 2011), but in the case of $S$. vortens, have never been documented (Sangmaneedet \& Smith 2000). Likewise, the cyst form has not yet been reported for S. salmonicida (host: salmon, trout), but a recent genomic survey identified in this parasite a Giardia lamblia cyst wall protein homologue as well as 2 enzymes involved in cyst wall assembly, glucosamine-6-phosphate isomerase and
UDP-N-acetylglucosamine pyrophosphorylase (Andersson et al. 2007). For S. meleagridis and S. salmonis, live trophozoites are also expelled into fresh faeces (Tojo \& Santamarina 1998, Philbey et al. 2002), and transmission of $S$. salmonicida trophozoites via skin lesions has been suggested (Poppe et al. 1992). The fact that $S$. vortens, S. salmonicida and $S$. meleagridis trophozoites are present in extra-intestinal systemic infections (Poppe et al. 1992, Paull \& Matthews 2001, Bailey et al. 2010) indicates that trophozoites are able to tolerate fluctuations in $\mathrm{O}_{2}$ tensions, $\mathrm{pH}$ and nutrition. Hence, it may be possible that $S$. vortens, S. salmonicida and $S$. meleagridis may not always require a cyst, with the trophozoite form instead responsible for transmission.

The transmission potential of a pathogen is commonly measured using the basic reproduction rate, $\mathrm{R}_{0}$, which represents the average number of secondary infections caused by an infected host during its lifetime. An $\mathrm{R}_{0}$ value $>1$ typically marks an epidemic outbreak, whereas if the calculated $R_{0}$ value is $<1$, the outbreak will become extinct (Woolhouse et al. 1997). The reliability of this epidemic threshold value, however, has recently been questioned as it can lead to erroneous conclusions regarding the infectivity of the pathogen and lead to inappropriate medical interventions for pathogen control (Breban et al. 2007). For example, $R_{0}$ is affected by heterogeneities in pathogen transmission rates between individuals in a given population (Woolhouse et al. 1997). Such variation in pathogen transmission can be partially explained by the Pareto Principle, a statistical pattern known as the 80/20 rule, as proposed by Woolhouse et al. (1997). Host-parasite systems commonly abide to the 80/20 rule, whereby approximately $80 \%$ of the parasites are harboured by $20 \%$ of the hosts. Thus these top $20 \%$ of hosts account for the bulk of the population's transmission potential. Such over-dispersal amongst hosts has been documented for the major soil-transmitted helminths (Ascaris lumbricoides, Trichuris trichiura, Necator americanus and Ancylostoma duodenale), schistosomes (Hotez et al. 2006) and malaria (Bousema et al. 2012).

Overall, the current study aimed to provide novel information on the life cycle of Spironucleus vortens, specifically its mode of transmission. Three distinct outcomes arose from this study: (1) We developed a statistical modelling technique to non-invasively estimate the degree of intestinal colonization by $S$. vortens using trophozoite faecal counts (based on a previous study by Tojo \& Santamarina 1998). This modelling method may be employed to replace cur- 
rent invasive methods of determining parasite burden, which involve euthanasia of fish in order to examine intestinal contents (see Whaley \& FrancisFloyd 1991, Sangmaneedet 1999, Jørgensen \& Sterud 2004). (2) The distribution of $S$. vortens within a host population was assessed using the non-invasive parasite quantification method to test the $80 / 20$ rule. (3) The role of the trophozoite in parasite transmission was examined using further faecal analysis (trophozoite survival), in vitro experiments (trophozoite tolerance to low $\mathrm{pH}$ ) and an antibody-based technique (in the search for putative $S$. vortens cysts).

\section{MATERIALS AND METHODS}

\section{Fish origin and general maintenance}

Juvenile angelfish Pterophyllum scalare ( $\mathrm{N}=168$, with an average length of ca. $5 \mathrm{~cm}$ ) were obtained from J\&K Aquatics (Taunton, UK). Fish were held individually for the duration of the experiment in 121 plastic tanks, each filled with dechlorinated water, an air supply and a plastic tank decoration for cover. Fish were maintained at $24 \pm 1^{\circ} \mathrm{C}$ with a $12: 12 \mathrm{~h}$ light:dark cycle and fed on a flake food (TetraMin) diet. Complete water changes were performed at least once per week in order to maintain good water quality.

\section{Parasite origins}

Spironucleus vortens (ATCC 50386). Trophozoite cultures were maintained in Keister's modified TYIS-33 medium according to a method adapted by Williams et al. (2012). Briefly, cultures were maintained at $24 \pm 1^{\circ} \mathrm{C}$ and sub-cultured at $48 \mathrm{~h}$ intervals by transferring $500 \mu \mathrm{l}$ of a mixed log-phase culture into $10 \mathrm{ml}$ of culture medium in $15 \mathrm{ml}$ screw capped Falcon tubes (Greiner Bio-one), leaving a $5 \mathrm{ml}$ head space. Cultures were monitored for microbial contamination by plating-out $100 \mu$ l of an exponentially growing culture on TYI-S-33 agar $(0.5 \% \mathrm{w} / \mathrm{v})$ and incubating under the above conditions for $5 \mathrm{~d}$.

Spironucleus vortens Sv1. An infected angelfish (identified by the presence of Spironucleus trophozoites in faeces, August 2011) that was bred in England was euthanized by overdose of MS-222 (SigmaAldrich). The entire intestinal tract was removed by severing at the anterior (just after the stomach) and posterior (at the anus) ends, and transferred to $10 \mathrm{ml}$ Keister's modified culture medium containing $0.5 \mathrm{mg}$ $\mathrm{ml}^{-1}$ penicillin and streptomycin, $5 \mathrm{mg} \mathrm{ml}^{-1}$ gentamycin and $1 \mathrm{mg} \mathrm{ml}^{-1}$ collistin sulphate (all from Sigma-Aldrich), and incubated for $72 \mathrm{~h}$ at $24^{\circ} \mathrm{C}$. This allowed trophozoites to migrate from the intestinal tract and into the surrounding culture medium through the open anterior and posterior ends of the gut. The organism was then sub-cultured 5 times as described above to allow an increase in trophozoite number and remove bacterial/fungal contamination. Finally, an unidentified ciliate contaminant derived from the fish gut was removed at room temperature $\left(20^{\circ} \mathrm{C}\right)$ by low speed centrifugation at $120 \times g(3 \mathrm{~min})$. The resulting pellet, containing the axenic Spironucleus isolate, was aseptically transferred to fresh culture medium and maintained as described above, without antibiotics. Freezer stocks of the new isolate were prepared after a total of 6 subcultures by suspending ca. $10^{6}$ cells $\mathrm{ml}^{-1}$ in $10 \%$ DMSO (v/v, SigmaAldrich) and cooling to $-80^{\circ} \mathrm{C}$ in an isopropanol bath at a controlled rate of $1^{\circ} \mathrm{C} \mathrm{min}^{-1}$. Vials of frozen cultures were then transferred to liquid $\mathrm{N}_{2}$ for long-term storage. Molecular identification of this new Spironucleus isolate (herein Sv1) was confirmed by PCR (see below). This new isolate was used alongside the ATCC strain in order to examine the occurrence of $S$. vortens cysts, as described below.

Spironucleus barkhanus (ATCC 50467). Trophozoites from a liquid $\mathrm{N}_{2}$ culture were thawed and incubated overnight in Keister's modified TYI-S-33 culture medium supplemented with $1 \mathrm{mg} \mathrm{l}^{-1}$ bile (bovine, sourced from a local slaughter house) at $20^{\circ} \mathrm{C}$, according to Sterud (1998). This organism was used a positive control for the PCR assay described below.

\section{Species-level identification of Spironucleus vortens using 16S rDNA PCR}

A new primer pair, SV-1f (5'-TGT GGG AGA CTG TGC TCT TG-3') and SV-1r: (5'-AGC ATA CTC CCC CAG GAA CT-3'), was designed to specifically amplify $158 \mathrm{bp}$ of the 16S rRNA gene of Spironucleus vortens beginning at positions 704 (SV-1f) and 842 (SV-1r) of the GenBank Accession Number U93085 S. vortens gene sequence (Keeling \& Doolittle 1997). These primers were designed using Primer3 (Rozen \& Skaletsky 2000), and their suitability for PCR was confirmed using OligoAnalyzer version 3.1 (Primer Quest@: www.idtdna.com/Scitools). The Spiro-1f/1r primer pair from Jørgensen \& Sterud (2004), which amplifies the 16S rRNA gene of most Spironucleus and Hexamita species, was employed as a positive 
control, producing an amplicon of $1431 \mathrm{bp}$. Total DNA was extracted from the $S v 1$ intestinal isolate, $S$. vortens ATCC strain (positive control), S. barkhanus ATCC strain (negative control) and $\mathrm{dH}_{2} \mathrm{O}$ (negative control) using the DNeasy Blood and Tissue Kit (Qiagen). Each $10 \mu \mathrm{l}$ PCR reaction consisted of $2 \mu \mathrm{l}$ of extracted DNA, $1 \mu \mathrm{l}$ of each primer $\left(10 \mathrm{pmol} \mathrm{ul}^{-1}\right.$, Eurofins), $0.1 \mu \mathrm{l}$ dNTPs (0.25 mM, Thermo Scientific), $0.6 \mu \mathrm{MgCl}_{2}$ (1.5 mM, Thermo Scientific), $1 \mu \mathrm{l}$ of PCR buffer (ABgene), $0.1 \mu$ l of Taq polymerase (0.05 U $\mu \mathrm{l}^{-1}$, Thermo Scientific) and $4.2 \mu \mathrm{l}$ of $\mathrm{dH}_{2} \mathrm{O}$. The PCR cycle was as follows: $95^{\circ} \mathrm{C}$ for $5 \mathrm{~min}, 95^{\circ} \mathrm{C}$ for $45 \mathrm{~s}$, $56^{\circ} \mathrm{C}$ for $1 \mathrm{~min}, 72^{\circ} \mathrm{C}$ for $2 \mathrm{~min}, 72^{\circ} \mathrm{C}$ for $4 \mathrm{~min}$ and finally held at $4^{\circ} \mathrm{C}$ in a 96-well thermal cycler (Applied Biosystems). The denaturing, annealing and elongation steps were repeated for 35 cycles. Gel electrophoresis of the PCR products $(2 \mu$ l each plus $1 \mu$ loading dye, Applied Biosystems) and $100 \mathrm{~kb}$ DNA ladder was performed on $1.5 \%(\mathrm{w} / \mathrm{v})$ agarose gels (Bioline) containing ethidium bromide (Invitrogen) with an applied voltage of $120 \mathrm{~V}$. Gels were then visualized and imaged using an UV transilluminator (GelDoc-It).

\section{Detection and quantification of Spironucleus vortens trophozoites in angelfish faecal pellets by light microscopy}

Fresh faecal pellets from angelfish were collected immediately after defecation from the bottom of clean plastic tanks using a plastic pipette. Preparation of faecal samples and estimation of parasite counts were conducted as previously described by Tojo \& Santamarina (1998). Whole faecal samples (ca. 0.5 to $1 \mathrm{~cm}$ in length) were placed on a glass slide with a drop of fresh dechlorinated water and compressed using a $22 \times 22 \mathrm{~mm}$ coverslip. These squash preparations were examined by light microscopy for the presence of Spironucleus vortens trophozoites, which were identified according to their length (5 to $10 \mu \mathrm{m})$, pyriform shape and characteristic rapid cell motility. The number of trophozoites per sample was qualitatively estimated by counting the number of parasites present for a $15 \mathrm{~min}$ period (400× magnification) until the whole sample had been examined (ca. 50 fields of view). Precise parasite counts were not possible due to intense trophozoite motility, which is a major characteristic employed in the identification of these organisms.

The PCR method described above, using the SV1f/1r primer pair, was used to confirm Spironucleus vortens in the faeces of angelfish. To test for the sen- sitivity of the method for non-invasive in vivo diagnosis, we analysed 17 angelfish faecal samples, from fish with varying degrees of Spironucleus infection (as determined by faecal counts, see below). Unconcentrated tank water $(1 \mathrm{ml})$ from a highly infected and uninfected fish was also tested for the presence of $S$. vortens, with fresh dechlorinated water used as an additional negative control.

\section{Comparison of infection prevalence and parasite density via faecal and intestinal trophozoite counts}

For non-invasive quantification of Spironucleus vortens trophozoites, 2 faecal samples (in order to account for variation in parasite shedding by the host over time, this was the maximum number of faecal samples produced by the angelfish in a single day) were collected from 20 fish. Later the same day, the fish were euthanized with an overdose of buffered MS-222 anaesthetic and dissected. The entire intestinal tract was removed and cut into 4 sections: anterior, anterior-middle, middle-posterior and posterior (according to Uldal \& Buchmann 1996), and the total number of $S$. vortens trophozoites in each section was estimated through the intestinal wall, as for the faecal samples (squash preparation of intestinal sections under 400× magnification).

In order to model the relationship between faecal and intestinal trophozoite densities, trophozoite counts derived from angelfish faeces and the 4 angelfish intestinal sections were first incorporated into a generalized linear mixed model to account for the repeated faecal sampling of 1 individual. Since animal ID and faecal sample number did not have a significant effect on the faecal trophozoite count, the averages of the 2 faecal samples were then used as dependent variables in a negative binomial general linear model (GLM) with intestinal trophozoite counts in the 4 different gut locations as independent variables. Models were reduced using a stepwise deletion process with an $\alpha$ level of 0.05 and evaluation of Akaike's information criterion (AIC) values to assess the significance of model terms and overall model fit. The only independent variable which significantly correlated with faecal counts was the posterior intestine. Hence, the final model utilized $\log _{10^{-}}$ transformed faecal (dependent variable) and posterior intestinal (independent variable) count data and the square root link function. The model assumptions were checked for normal distribution (visual examination of histograms and Q-Q plots), heteroscedasticity (Fligner-Killeen test, $p>0.05$ ), 
overdispersion (theta $=\sim 1$ ) and proportion of variability in the dataset that is accounted for by the model (pseudo- $\mathrm{r}^{2} \geq 0.7$ ). The final model was then used to predict the number of trophozoites in the posterior gut based on parasite counts in faeces.

\section{Pareto Principle (80/20 rule)}

The Spironucleus vortens trophozoite faecal quantification method described above was used to test the Pareto Principle that ca. $80 \%$ of the effects come from $20 \%$ of the causes. Hosts $(\mathrm{N}=168$, including uninfected individuals) were ranked from most to least infected according to semi-quantitative trophozoite faecal counts and the proportions of host ( 0 to 1 , with 0.05 increments) plotted against the corresponding proportions of parasites. From the resulting line chart, the expected proportion of parasites, or transmission potential, was extrapolated from the top $20 \%$ of most infected individuals.

\section{Survival of Spironucleus vortens trophozoites in faecal pellets}

Fresh faecal samples were collected from angelfish immediately after defecation, transferred into 11 plastic containers and incubated at $24^{\circ} \mathrm{C}$ for 0 (immediately counted after defecation), 1, 2, 3, 14 or $36 \mathrm{~d}$ in dechlorinated water $(\mathrm{N}=28$ faecal samples per time point). After incubation, samples were examined and the number of Spironucleus vortens trophozoites estimated as described above.

\section{Survival of Spironucleus vortens trophozoites at varying $\mathrm{pH}$ in vitro}

Survival of Spironucleus vortens in acidic pH was examined to determine whether trophozoites are able to survive transmission through the stomach. Log-phase $S$. vortens trophozoites from in vitro cultures (ATCC and Sv1 strains) were inoculated into the wells of a 12-well plate to a final cell density of 2 $\times 10^{6}$ to $3 \times 10^{6}$ cells well $^{-1}$ (counted by fixation of a $10 \mu \mathrm{l}$ sample from a homogenous cell suspension in $1.5 \% \mathrm{v} / \mathrm{v}$ formaldehyde using an Improved Neubauer haemocytometer prior to well inoculation). Each well contained $2 \mathrm{ml}$ of culture medium at different $\mathrm{pH}$ levels: $\mathrm{pH} 3,4,5,6,7$ or $2 \mathrm{ml}$ of distilled water $(2$ replicates of each $\mathrm{pH}$ per parasite strain). Trophozoite motility was used to monitor cell vigour using light microscopy (200× magnification). Cells were incubated on a shaker between observations (50 rpm) to mimic the motions of stomach peristalsis and observed every hour for $3 \mathrm{~h}$. The transit time of food through the stomach in humans is ca. $2 \mathrm{~h}$, whilst that of rats is far quicker $(75 \%$ of stomach contents emptied in 15 min; Kararli 1995, Bodé et al. 2004). Hence, the transit time of food through an angelfish stomach is likely to be within the maximum incubation period selected of $3 \mathrm{~h}$. Cell motility was ranked as follows: $(-)$ immotile cells, $(+)$ twitching cells $(++)$, semi-motile/slow-moving cells and (+++) cells with normal/healthy motility.

\section{Putative Spironucleus vortens cysts}

Encystment of Spironucleus vortens in vitro. To investigate whether $S$. vortens encystment could be induced, 4 methods were tested that are based on successful encystment protocols for the related diplomonad Giardia and other Spironucleus spp. These were as follows: (1) starvation: post-stationary phase cultures (i.e. $>72 \mathrm{~h}$ ) incubated in phosphate-buffered saline (pH 7.2) or distilled water (Uldal 1996); (2) Giardia encystment medium: $S$. vortens trophozoites (ATCC and $S v 1$ strains) were grown to late log phase in culture medium, before being transferred to encystment medium, consisting of culture medium supplemented with $1 \mathrm{M} \mathrm{NaOH}$ (Sigma-Aldrich), $0.25 \mathrm{mg} \mathrm{ml}^{-1}$ bovine bile (obtained from local slaughter house) and $5 \mathrm{mM}$ lactic acid (Sigma-Aldrich, Gillin et al. 1989); (3) bile salts: taurocholic acid and glycocholic acid (both from Sigma-Aldrich) in culture medium $(1,2,4,8$ and $16 \mathrm{mM})$; (4) fatty acids: oleic acid and myristic acid (both from Sigma-Aldrich), in culture medium $(2,1,0.5,0.25$ and $0.125 \mathrm{mM})$. Approximately $10^{6} \mathrm{~S}$. vortens trophozoites were incubated with all treatments for $72 \mathrm{~h}$ in 12-well plates ( $2 \mathrm{ml}$ volume for each treatment) and examined by light microscopy every $24 \mathrm{~h}$ for the presence of putative cysts using the trypan blue $(0.4 \% \mathrm{w} / \mathrm{v}$, SigmaAldrich) exclusion assay to identify viable cells.

Morphological identification of putative Spironucleus vortens cysts in vivo. There are no commercially available cyst-specific stains for the identification of Spironucleus. However, Januschka et al. (1988) showed that the cysts of S. muris and Giardia microti have similar cyst wall protein compositions. Therefore, the GiardiaCel kit (Cellabs) was used to examine angelfish faecal samples for the presence of Spironucleus cysts. Fresh $(\mathrm{n}=10)$ and 2 wk old $(\mathrm{n}=$ 10) faecal samples (the latter incubated in aquarium 
water in closed Eppendorf tubes) were collected from angelfish and fixed, permeabilized and stained according to the manufacturer's instructions. The kit contained a Giardia-specific cyst wall primary antibody, which binds to CWP1, a protein component of the Giardia cyst wall (Chatterjee et al. 2010). To visualize the cyst, a fluorescent fluorescein isothiocyanate (FITC)-tagged anti-mouse secondary antibody was used ( $\lambda_{\text {ex }} 490 \mathrm{~nm}, \lambda_{\text {em }} 530 \mathrm{~nm}$ ). Cells were imaged at $200 \times$ and $600 \times$ magnification using confocal microscopy (Leica TCS SP2 AOBS). A Giardia-positive slide, supplied with the kit, was used as a positive control. S. vortens trophozoites (ATCC strain) from $5 \mathrm{~d}$ and $2 \mathrm{wk}$ old in vitro cultures were also examined in the same way.
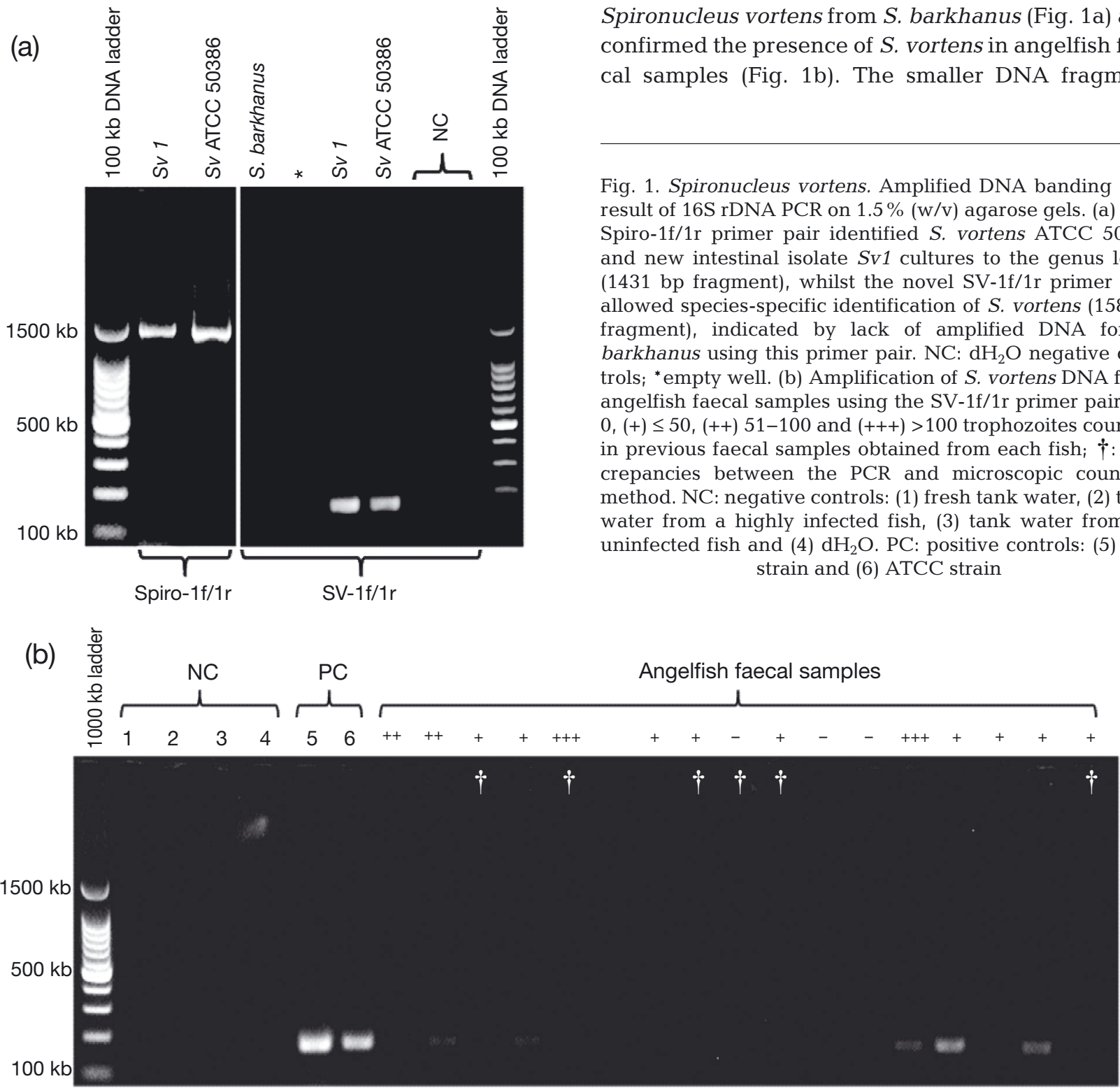

\section{Statistical analysis}

All statistical analyses described above were conducted using R (version 2.15.1; R Development Core Team 2012). To compare faecal and intestinal trophozoite counts, mean density was used, and is defined as the mean number of parasites counted per sample, with zero counts included.

\section{RESULTS}

\section{Identification of Spironucleus vortens trophozoites in fresh angelfish faecal pellets}

The SV-1f/1r primer pair specifically distinguished Spironucleus vortens from $S$. barkhanus (Fig. 1a) and confirmed the presence of $S$. vortens in angelfish faecal samples (Fig. 1b). The smaller DNA fragment

Fig. 1. Spironucleus vortens. Amplified DNA banding as a result of $16 \mathrm{~S}$ rDNA PCR on $1.5 \%$ (w/v) agarose gels. (a) The Spiro-1f/1r primer pair identified S. vortens ATCC 50386 and new intestinal isolate $S_{V} 1$ cultures to the genus level (1431 bp fragment), whilst the novel SV-1f/1r primer pair allowed species-specific identification of $S$. vortens (158 bp fragment), indicated by lack of amplified DNA for $S$. barkhanus using this primer pair. $\mathrm{NC}$ : $\mathrm{dH}_{2} \mathrm{O}$ negative controls; ${ }^{*}$ empty well. (b) Amplification of $S$. vortens DNA from angelfish faecal samples using the SV-1f/1r primer pair. (-) $0,(+) \leq 50,(++) 51-100$ and $(+++)>100$ trophozoites counted in previous faecal samples obtained from each fish; $\uparrow$ : discrepancies between the PCR and microscopic counting method. NC: negative controls: (1) fresh tank water, (2) tank water from a highly infected fish, (3) tank water from an uninfected fish and (4) $\mathrm{dH}_{2} \mathrm{O}$. PC: positive controls: (5) $\mathrm{Sv} 1$ strain and (6) ATCC strain 


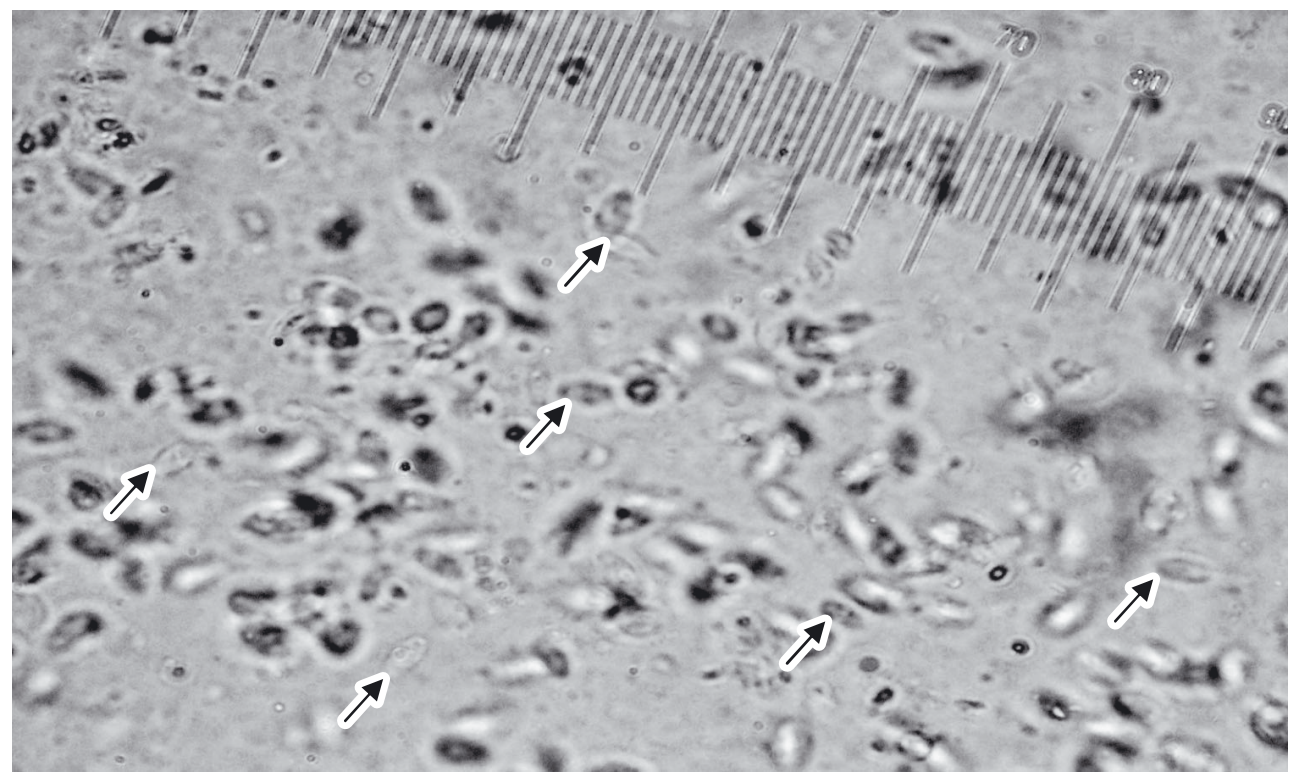

Fig. 2. Spironucleus sp. Numerous (ca. 70) motile trophozoites in the faeces of an angelfish. Note the characteristic length (ca. $5-10 \mu \mathrm{m})$ and pyriform shape of parasites, indicated by arrows $(400 \times$ magnification, 1 eye piece unit $=2.5 \mu \mathrm{m})$

generated from the new primer pair was easy to amplify, and therefore the test was more sensitive than the previously documented Spiro-1f/1r primer pair (see Jørgensen \& Sterud 2004; data not shown), which only identifies Spironucleus to the genus level.

Single-celled, pyriform-shaped, highly motile flagellates with rapid motion were observed at 400x magnification mostly towards the centre of the faecal squash preparations (Fig. 2). These organisms were ca. 5 to $10 \mu \mathrm{m}$ in length, abundant and were found freely swimming amongst the faecal matter. Numerous intracellular inclusions, putative vacuoles and/or bacteria, were observed within these organisms, which have been characteristically observed in Spironucleus trophozoites (Poynton \& Sterud 2002). These cytoplasmic inclusions are less numerous in laboratory cultured isolates.

The mean density of Spironucleus vortens trophozoites in faecal samples of all fish $(\mathrm{N}=168)$ was 68 , with a range of 1 to 1322. Differences were observed in the sensitivity of the molecular and microscopic approaches for identifying $S$. vortens trophozoites. This is illustrated in Fig. 1b, whereby no $S$. vortens DNA amplification was apparent in faecal samples of fish previously deemed to be highly infected with $S$. vortens (>100 trophozoites counted) by the microscopic method. However, not enough replicates were performed in order to directly compare the accuracy of the microscopic and molecular methods.

\section{Correlation between faecal and intestinal trophozoite counts}

The variation between faecal counts taken from the same fish over time is illustrated in Fig. 3. The largest difference between 2 faecal samples from the same host was 685 trophozoites, with a range of 135 to 820 parasites counted. However, this is an extreme example indicating an occasional outlier that was infrequently encountered. The prevalence of infection in the intestinal tract of angelfish ( $\mathrm{N}=20)$ was $100 \%$, with a mean density of 3096 , ca. $46 \times$ greater than that

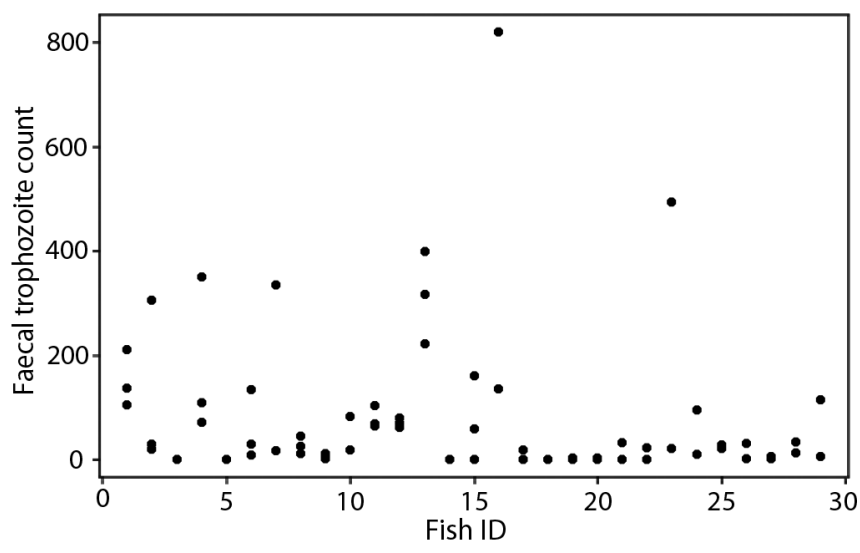

Fig. 3. Spironucleus sp. Variability in the total number of trophozoites observed per faecal sample $(n \geq 2)$ at $\times 400$ magnification for each fish (29 in total) 


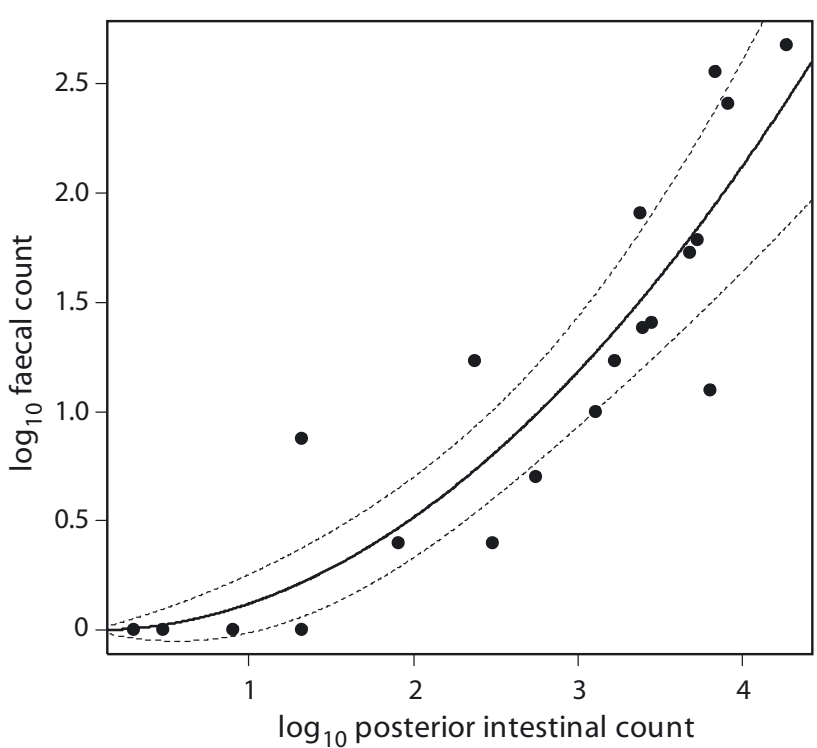

Fig. 4. Spironucleus vortens. $\log _{10}$-transformed faecal trophozoite count vs. posterior intestinal trophozoite count. Continuous line: predicted values of posterior counts for a given faecal count, derived from a negative binomial general linear model $(Z=3.979, \mathrm{p}<0.001$, theta $=0.308$ and pseudo- $\mathrm{R}^{2}=0.7$ ); this prediction curve was overlaid on top of a scatterplot of the actual count data. Dashed lines: upper and lower SEs of the model

of faecal samples (mean density 68, see previous section), with a range of 1 to 18430 in the posterior section (the intestinal section which had the highest parasite load). There was a significant positive correlation between average faecal trophozoite counts and posterior intestinal trophozoite counts (negative binomial GLM, $Z=3.979, \mathrm{p}<0.001, \mathrm{AIC}=44.60$, pseudo- $\mathrm{R}^{2}=0.7$; Fig. 4). Although individual faecal counts, i.e. count 1 or count 2 , when incorporated individually into the model, also significantly correlated with posterior intestinal counts, the overall fit of both of these models was reduced with regards to the proportion of variability in the dataset that was accounted for by the models (pseudo- $\mathrm{R}^{2}=0.58$ and 0.50 for count 1 and count 2, respectively). Hence, obtaining the average of at least 2 faecal counts greatly increases reliability when predicting posterior intestinal trophozoite numbers.

\section{Aggregation of Spironucleus vortens infection in angelfish}

Fig. 5 shows that $20 \%$ of angelfish hosts harbour approximately $83 \%$ of parasites, and thus transmission potential of the population. Of the total host population, $85 \%$ harboured $100 \%$ of the parasites.

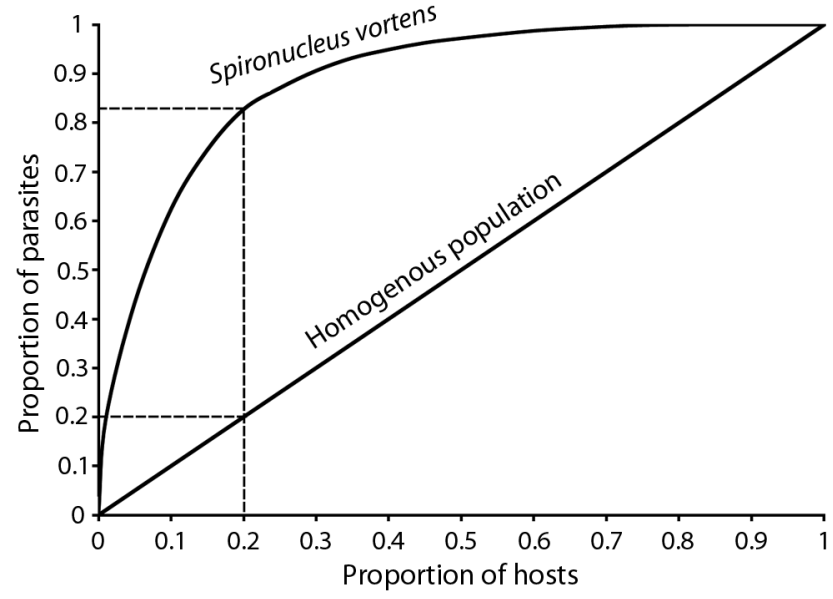

Fig. 5. Pterophyllum scalare infected by Spironucleus vortens. Fitted line plot (continuous line) of the proportion of hosts $(\mathrm{N}=168)$, ranked from most to least infected with $S$. vortens, against the proportion of parasites in order to test the Pareto Principle (or 80/20 rule) of parasite aggregation in a host population. The distribution of a homogeneous population is also indicated. Dashed lines: expected proportion of parasites present in $20 \%$ of the hosts

\section{Survival of Spironucleus vortens trophozoites in faeces and varying $\mathrm{pH}$}

Spironucleus vortens trophozoites survived in the angelfish faecal pellets for a minimum of $36 \mathrm{~d}$ (the final time point examined during this experiment). Between 1 and 16 S. vortens trophozoites were observed in the $36 \mathrm{~d}$ old faecal samples, with a mean density of 2 . This was a decline of $90 \%$ compared to the Day 0 samples (mean density 19.2, range 1 to 130 ).

Spironucleus vortens trophozoites (both the Sv1 and ATCC strains) survived for a minimum of $3 \mathrm{~h}$ in culture medium at $\mathrm{pH} 5$ and 7 indicated by motility of the organisms. Parasite motility in $\mathrm{dH}_{2} \mathrm{O}$ and at $\mathrm{pH} 6$ was reduced to twitching (ATCC strain) or no motility (Sv1 strain) after $3 \mathrm{~h}$. Motility of the Sv1 new intestinal isolate also diminished greatly following $3 \mathrm{~h}$ incubation in pH 5 and 7 culture media. However, for cells incubated in culture medium at $\mathrm{pH} \leq 4$, cell motility immediately ceased for both strains and trophozoites became irregular in morphology, eventually resulting in lysis of cells (Table 1).

\section{Attempted induction and identification of Spironucleus vortens cysts}

No viable cyst-like structures were observed after incubation in any of the 4 cyst-induction media tested. After incubation, there was a marked increase 
Table 1. Spironucleus vortens. Survival of $S$. vortens (ATCC and $\left.S_{V} 1\right)$ trophozoites over time in culture medium of varying $\mathrm{pH}$ and distilled water, indicated by $(+++)$ healthy motility, (++) semi-motile, $(+)$ twitching motility and (-) immotile cells. Cells were shaken between each observation

\begin{tabular}{|lccccc|}
\hline Time (h) & pH 4 & pH 5 & pH 6 & pH 7 & $\mathrm{dH}_{2} \mathrm{O}$ \\
\hline ATCC strain & & & & & \\
0 & - & +++ & +++ & +++ & +++ \\
1 & - & +++ & +++ & +++ & +++ \\
2 & - & +++ & ++ & +++ & ++ \\
3 & - & +++ & + & +++ & + \\
$S_{V}$ 1 strain & & & & & \\
0 & - & +++ & +++ & +++ & +++ \\
1 & - & ++ & ++ & ++ & +++ \\
2 & - & + & + & ++ & ++ \\
3 & - & + & - & + & + \\
\hline
\end{tabular}

in lysed cell debris (i.e. aggregations of organelles) and trypan blue was taken up by all remaining intact cellular structures, indicating dead or dying cells. Fig. 6a shows the characteristic staining of the Giardia cyst wall, which was used as a positive control in the search for putative $S$. vortens cysts. No Spironucleus cysts were identified in any of the angelfish faecal samples. There was a high degree of crossreactivity of the Giardia cyst wall antibody with other matter found within the faecal samples, but no stained structures were observed that were similar in morphology to Giardia and $S$. muris cysts (see Januschka et al. 1988). Interestingly, however, S. vortens trophozoites from $5 \mathrm{~d}$ old and $2 \mathrm{wk}$ old cultures were positively labelled with this antibody (Fig. 6b-d). Unlike the Giardia cysts, intact trophozoites were labelled only around the periphery of the cell.
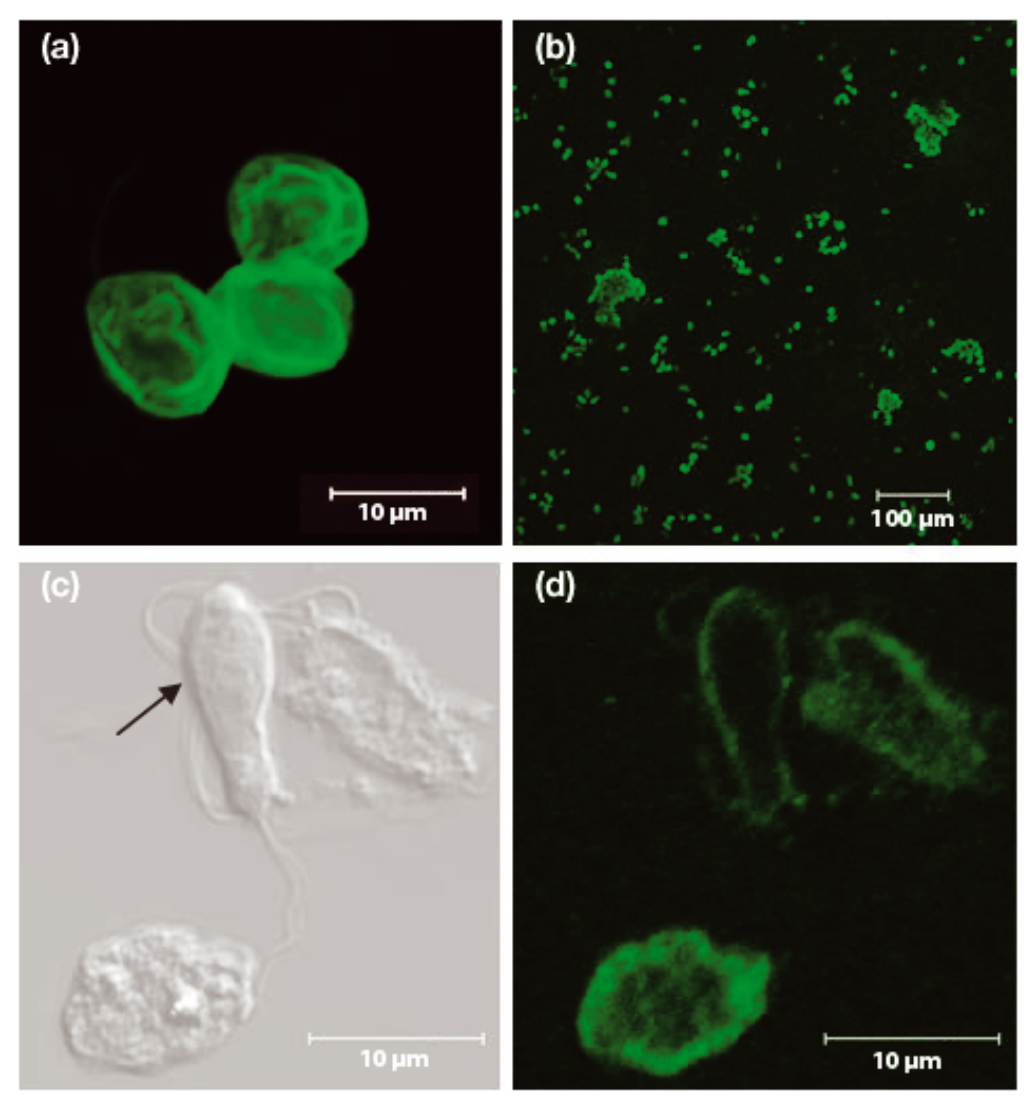

Fig. 6. Giardia sp. and Spironucleus vortens. Staining of diplomonad trophozoites (ATCC strain) with GiardiaCel antibody. (a) Giardia cysts (positive control), (b) 2 wk old $S$. vortens culture, (c) differential interference contrast image of $5 \mathrm{~d}$ old $S$. vortens culture (arrow indicates an intact trophozoite with visible flagella) and (d) fluorescence FITC image of (c). The debris at the bottom left hand side of images (c) and (d) is a non-viable (dead) trophozoite from the culture

\section{DISCUSSION}

Using non-invasive methods, this study presents compelling evidence to support trophozoite- (rather than cyst-) mediated transmission of Spironucleus vortens in angelfish.

\section{Non-invasive semi-quantitative estimation of Spironucleus vortens intestinal load}

The characteristic rapid motility of Spironucleus vortens, as well as its pyriform shape and typical length of ca. 5 to $10 \mu \mathrm{m}$, allowed easy identification of $S$. vortens from squash preparations of angelfish intestinal sections and faecal pellets during this study. The presence of parasites in samples was confirmed using novel SV-1f/1r primers, which provided species level identification of $S$. vortens. Intestinal dissections revealed that all fish examined $(\mathrm{N}=20)$ were infected with $S$. vortens. Density of infection was greatest in the posterior intestine, which is comparable with previous observations by Poynton et al. (1995) and Sangmaneedet (1999).

As a non-invasive method of quantifying infection, faecal trophozoite counts were used as a tool to predict the degree of intestinal colonization by 
Spironucleus vortens. Faecal trophozoite counts have been used previously by Tojo \& Santamarina (1998) as a means of estimating $S$. salmonis infections in salmonids. The current study confirms the feasibility of employing faecal trophozoite counts to estimate the degree of intestinal colonization, with faecal counts correlating significantly with trophozoite counts observed in the posterior intestine of angelfish. It is important to note, however, that the mean density of posterior intestinal trophozoite counts was $46 \times$ greater than the faecal trophozoite counts. Hence, care must be taken whilst interpreting faecal samples where no trophozoites are detected for both the microscopic and molecular identification methods, as this may be due to human counting error, reduced parasite motility in the sample or differential parasite shedding by the host over time. The latter is true of Giardia, for which periodicity in cyst excretion by the host has been reported (McGlade et al. 2003). We therefore recommend that a minimum of 2 faecal samples are analysed from the same fish in order to estimate intestinal colonization.

\section{Aggregation of Spironucleus vortens in angelfish}

Parasite aggregation of Spironucleus vortens in hosts was investigated using data from faecal trophozoite counts. In the current study, angelfish $S$. vortens aggregation matched the $80 / 20$ rule, with $20 \%$ of the hosts harbouring $83 \%$ of parasites. These highly infected hosts, termed 'supershedders', account for the bulk of the transmission potential (see Lloyd-Smith et al. 2005). Woolhouse et al. (1997) observed a similar aggregation pattern for the trypanosomatid and apicomplexan protozoan parasites Leishmania and Plasmodium, respectively. The same authors suggested that control measures should focus on this $20 \%$ core of highly infected individuals. Hence, the non-invasive trophozoite quantification method developed during the current study could be applied in aquaculture to identify and treat the top $20 \%$ of infected individuals in order to efficiently control disease outbreaks. This would be especially useful for broodstock or other highly prized angelfish and discus.

\section{Transmission potential of Spironucleus vortens trophozoites}

A small proportion of expelled Spironucleus vortens trophozoites (ca. $10 \%$ of the number recovered from fresh faecal samples) survived for $36 \mathrm{~d}$ in angelfish faecal pellets. At this stage, it is not clear whether individual trophozoites survived for this period or whether cell division occurred; however, this is the first observation of prolonged survival of diplomonad trophozoites in host faeces. This suggests that $S$. vortens trophozoites are able to use substrates present in the faeces and withstand fluctuations in nutrients, temperature and $\mathrm{O}_{2}$ tensions for long-term survival. Millet et al. (2010) found that $S$. vortens consumes $\mathrm{O}_{2}$ at a rate of $62 \mathrm{nmol} \mathrm{min} \mathrm{m}^{-1}$ per $10^{7}$ cells in a closed reaction vessel, suggesting a highly effective $\mathrm{O}_{2}$ scavenging mechanism. $S$. vortens is only capable of surviving in water for a limited time (10 h, in vitro study; Millet et al. 2011), a finding which was confirmed during the current study whereby trophozoite motility was dramatically reduced after $3 \mathrm{~h}$. Hence, faecal matter is likely to confer a substantial degree of protection for the trophozoite against the external environment. For example, the presence of $\mathrm{O}_{2}$-consuming gut bacteria and saprophytic organisms will result in the removal of $\mathrm{O}_{2}$ from faeces, generating a microaerobic habitat. Indeed, trophozoites were mainly found towards the centre of faecal samples where $\mathrm{O}_{2}$ tensions would be at their lowest (pers. obs.). S. salmonis trophozoites have also been observed in the faeces of salmonids by Tojo \& Santamarina (1998); however, prolonged survival of these trophozoites in trout faeces was not investigated in this previous study. The fact that $S$. vortens and $S$. salmonis cluster together in 16S rDNA phylogenies suggests that they are closely related, and may explain the similarities observed in the presence of trophozoites in the faeces of their hosts (Fard et al. 2007).

Kent et al. (1992) showed that trophozoites of a marine diplomonad, later described as Spironucleus salmonicida (Jørgensen \& Sterud 2006), could be transmitted via water-borne exposure and gavage of Atlantic and Chinook salmon. In vitro studies, also conducted by Kent et al. (1992), revealed that these trophozoites survived poorly in freshwater $(<5 \mathrm{~min})$ and seawater $(<4 \mathrm{~h})$; thus it was suggested that host mucus or faeces confers protection to the trophozoite from osmotic stress. In the same study, no S. salmonicida cysts were detected (Kent et al. 1992). Hence, transmission of $S$. salmonicida and $S$. vortens is likely to be similar, directly facilitated by trophozoites.

As Spironucleus vortens trophozoites can survive for extended periods in angelfish faecal pellets, they potentially represent a transmission stage to new hosts, but if ingested they would have to withstand the acidic $\mathrm{pH}$ of the stomach. $S$. vortens trophozoites generally remained motile at $\mathrm{pH} 5-7$, but motility 
instantly ceased at $\mathrm{pH} \leq 4$, eventually resulting in cell lysis. Reduced motility of trophozoites over time at $\mathrm{pH} 6$, as compared to that at $\mathrm{pH} 5$ and 7 , is intriguing. This may be explained by the possible presence of $\mathrm{pH}$-sensitive metabolic or antioxidant enzymes in $S$. vortens, and requires further investigation. The stomach $\mathrm{pH}$ of freshwater angelfish has not been well characterised; however, that of Holacanthus passer, a marine angelfish species, has been reported to range from 2 to 5 (Martínez-Díaz \& Pérez-España 1999). In another cichlid, Oreochromis niloticus, stomach $\mathrm{pH}$ levels range from 0.9 to 7 (Getachew 1989), being more acidic after feeding due to the secretion of gastric fluids for digestion. Hence, at the more alkaline end of the scale it may be plausible that $S$. vortens trophozoites can survive passage through the stomach. Indeed, Spironucleus trophozoites have previously been documented in the stomach of hosts, including mice (Fain et al. 2008), Siamese fighting fish (O'Brien et al. 1993) and angelfish (Sangmaneedet 1999), with that of mice being confirmed by molecular identification. As some fish, including angelfish (pers. obs.) are coprophagic (Bailey \& Robertson 1982), it is highly likely that $S$. vortens trophozoites would be readily consumed and, given the appropriate conditions, may survive passage to the intestine. Indeed, Sangmaneedet (1999) showed that oral infection of angelfish with $S$. vortens trophozoites resulted in intestinal colonization by this parasite.

\section{Spironucleus vortens encystment?}

It has been proposed that cyst-like structures confer long-term survival of Spironucleus species outside the host (Woo \& Poynton 1995). However, despite various methods being employed during the current study to induce encystment in S. vortens, no viable cyst-like structures were identified. Hence, under the conditions described, neither starvation (in spent culture medium and water) nor putative chemical triggers of encystment, e.g. bile and lactic acid, induced encystment of $S$. vortens in vitro. The latter chemical triggers have previously been documented as encystment-induction factors of the related diplomonads S. salmonis and Giardia intestinalis (see Gillin et al. 1989, Uldal 1996). For some protists, e.g. Entamoeba histolytica, it has been notoriously difficult to induce encystment in vitro, despite the confirmed presence of cysts in vivo. For E. histolytica, reactive $\mathrm{O}_{2}$ species from the host immune system or intestinal microbiota have been suggested as poten- tial triggers of encystment, a stress factor that may not be present to the same degree in vitro (AguilarDíaz et al. 2010). As S. vortens occupies a similar niche to E. histolytica (i.e. posterior intestine), this may explain the failure to induce encystment in vitro during this study. As a result, angelfish faecal samples were also analysed for the presence of putative $S$. vortens cysts. Due to morphological similarities between the cyst walls of $G$. muris and $S$. muris (see Poppe et al. 1992), a cyst wall antibody from $G$. intestinalis was employed to identify $S$. vortens cysts in angelfish faeces. Again, no such structures were identified, either indicating a lack of cysts or nonspecificity of the Giardia antibody with $S$. vortens cysts. Interestingly, however, $S$. vortens trophozoites (ATCC strain) were found to be positively labelled with the antibody. The antibody has been previously shown not to react with Giardia trophozoites (WB strain, in culture since 1978; Chatterjee et al. 2010), which suggests that either the cell membrane of $S$. vortens trophozoites shares a similar epitope with the Giardia cyst wall, perhaps as a constituent of a protective pellicle, or that these trophozoites were in the process of encysting. Completion of the genome sequence of $S$. vortens will provide further insight into the presence or absence of encystment-specific genes in this organism.

\section{CONCLUSION}

In conclusion, our results call into question the existence of a cyst stage in the life cycle of Spironucleus vortens. This is especially relevant in aquaculture where host density, and therefore transmission potential via the faecal-oral route, is high. The noninvasive methods (faecal counts and 16S rDNA PCR) employed in this study require further investigation to determine their applicability in aquaculture for reliable diagnosis of $S$. vortens infection and subsequent control of spironucleosis. Prolonged survival of $S$. vortens trophozoites in faeces outside the host is a novel discovery that requires further physiological elucidation in terms of the capacity of the organism to tolerate high fluctuations in $\mathrm{O}_{2}$ tensions and nutrition status.

Acknowledgements. We thank E. Sherrard-Smith and L. Joshi for their advice on analysis of the 80/20 rule and primer design, respectively. The work was funded by an Engineering and Physical Sciences Research Council (EPSRC) Case Studentship (EP/H501118/1) in collaboration with NEEM Biotech Ltd. to C.F.W. 


\section{LITERATURE CITED}

Aguilar-Díaz H, Díaz-Gallardo M, Laclette JP, Carrero JC (2010) In vitro induction of Entamoeba histolytica cystlike structures from trophozoites. PLoS Negl Trop Dis 4: e607

Andersson JO, Sjögren AM, Horner DS, Murphy CA and others (2007) A genomic survey of the fish parasite Spironucleus salmonicida indicates genomic plasticity among diplomonads and significant lateral gene transfer in eukaryote genome evolution. BMC Genomics 8:51-76

Ankarklev J, Jerlström-Hultqvist J, Ringqvist E, Troell K, Svärd SG (2010) Behind the smile: cell biology and disease mechanisms of Giardia species. Nat Rev Microbiol 8:413-422

Bailey TG, Robertson DR (1982) Organic and caloric levels of fish feces relative to its consumption by coprophagous reef fishes. Mar Biol 69:45-50

Bailey C, Kramer J, Mejia A, MacKay J, Mansfield KG, Miller AD (2010) Systemic spironucleosis in two immunodeficient rhesus macaques (Macaca mulatta). Vet Pathol 47:488-494

- Biagini GA, McIntyre PS, Finlay BJ, Lloyd D (1998) Carbohydrate and amino acid fermentation in the free-living primitive protozoan Hexamita sp. Appl Environ Microbiol 64:203-207

> Bodé S, Dreyer M, Greisen G (2004) Gastric emptying and small intestinal transit time in preterm infants: a scintigraphic method. J Pediatr Gastroenterol Nutr 39: 378-382

Bousema T, Griffin JT, Sauerwein RW, Smith DL and others (2012) Hitting hotspots: spatial targeting of malaria for control and elimination. PLoS Med 9:e1001165

Breban R, Vardavas R, Blower S (2007) Theory versus data: how to calculate $\mathrm{R}_{0}$ ? PLoS ONE 2:e282

> Chatterjee A, Carpentieri A, Ratner DM, Bullitt E, Costello CE, Robbins PW, Samuelson J (2010) Giardia cyst wall protein 1 is a lectin that binds to curled fibrils of the GalNAc homopolymer. PLoS Pathog 6:e1001059

Fain MA, Karjala Z, Perdue KA, Copeland MK, Cheng LI, Elkins WR (2008) Detection of Spironucleus muris in unpreserved mouse tissue and faecal samples by using a PCR assay. J Am Assoc Lab Anim Sci 47:39-43

FAO (Food and Agriculture Organization of the United Nations) (2005) Fisheries and aquaculture topics. Ornamental fish. Topics fact sheets. Fisheries and Aquaculture Department, FAO, Rome. Available at www.fao. org/fishery/topic/13611/en (accessed 8 January 2013)

FAO (2010) The state of world fisheries and aquaculture 2010. Fisheries and Aquaculture Department, FAO, Rome. Available at www.fao.org/docrep/013/i1820e/ i1820e00.htm (accessed 22 April 2013)

Fard MRS, Jørgensen A, Sterud E, Bleiss W, Poynton SL (2007) Ultrastructure and molecular diagnosis of Spironucleus salmonis (Diplomonadida) from rainbow trout Oncorhynchus mykiss in Germany. Dis Aquat Org 75 : 37-50

FDA (US Food and Drug Administration) (2012) Safety $\&$ health. Antimicrobial resistance. Animal husbandry and disease control: aquaculture. FDA, Silver Spring, MD. Available at www.fda.gov/AnimalVeterinary/Safety Health/AntimicrobialResistance/ucm082099.htm (accessed 8 January 2013)

Getachew T (1989) Stomach pH, feeding rhythm and ingestion rate in Oreochromis niloticus L. (Pisces: Cichlidae) in
Lake Awasa, Ethiopia. Hydrobiologia 174:43-48

Gillin FD, Boucher SE, Rossi SS, Reiner DS (1989) Giardia lamblia: the roles of bile, lactic acid, and $\mathrm{pH}$ in the completion of the life cycle in vitro. Exp Parasitol 69:164-174

Hotez PJ, Bundy DAP, Beegle K, Brooker S and others (2006) Helminth infections: soil-transmitted helminth infections and schistosomiasis. In: Jamison DT, Breman JG, Measham AR, Alleyne G and others (eds) Disease control priorities in developing countries, 2nd edn. World Bank, Washington, DC, p 467-482

Januschka MM, Erlandsen SL, Bemrick WJ, Schupp DG, Feely DE (1988) Comparison of Giardia microti and Spironucleus muris cysts in the vole: an immunocytochemical, light and electron microscopic study. J Parasitol 74:452-458

Jørgensen A, Sterud E (2004) SSU rRNA gene sequence reveals two genotypes of Spironucleus barkhanus (Diplomonadida) from farmed and wild Arctic charr Salvelinus alpinus. Dis Aquat Org 62:93-96

> Jørgensen A, Sterud E (2006) The marine pathogenic genotype of Spironucleus barkhanus from farmed salmonids redescribed as Spironucleus salmonicida n. sp. J Eukaryot Microbiol 53:531-541

Jørgensen A, Sterud E (2007) Phylogeny of Spironucleus (Eopharyngia: Diplomonadida: Hexamitinae). Protist 158: 247-254

Kararli TT (1995) Comparison of the gastrointestinal anatomy, physiology, and biochemistry of humans and commonly used laboratory animals. Biopharm Drug Dispos 16:351-380

Keeling PJ, Doolittle WF (1997) Widespread and ancient distribution of a noncanonical genetic code in diplomonads. Mol Biol Evol 14:895-901

Kent ML, Ellis J, Fournie JW, Dawe SC, Bagshaw JW, Whitaker DJ (1992) Systemic hexamitid (Protozoa: Diplomonadida) infection in seawater pen-reared Chinook salmon Oncorhynchus tshawytsha. Dis Aquat Org 14:81-89

Kolisko M, Silbermann JD, Cepicka I, Yubuki N and others (2010) A wide diversity of previously undetected freeliving relatives of diplomonads isolated from marine/ saline habitats. Environ Microbiol 12:2700-2710

> Lloyd D, Harris JC, Maroulis S, Wadley R and others (2002) The 'primitive' microaerophile Giardia intestinalis (syn. lamblia, duodenalis) has specialized membranes with electron transport and membrane-potential-generating function. Microbiology 148:1349-1354

- Lloyd-Smith JO, Schreiber SJ, Kopp PE, Getz WM (2005) Superspreading and the effect of individual variation on disease emergence. Nature 438:355-359

> Martínez-Díaz SF, Pérez-España H (1999) Feasible mechanisms for algal digestion in the king angelfish. J Fish Biol 55:692-703

McGlade TR, Robertson ID, Elliot AD, Thompson RCA (2003) High prevalence of Giardia detected in cats by PCR. Vet Parasitol 110:197-205

Millet CO, Cable J, Lloyd D (2010) The diplomonad fish parasite Spironucleus vortens produces hydrogen. J Eukaryot Microbiol 57:400-404

> Millet CO, Lloyd D, Coogan MP, Rumsey J, Cable J (2011) Carbohydrate and amino acid metabolism of Spironucleus vortens. Exp Parasitol 129:17-26

Noga EJ (2010) Methods for diagnosing fish diseases. In: Noga EJ (ed) Fish disease: diagnosis and treatment. Wiley-Blackwell, Ames, IA, p 4-81 
O'Brien GM, Ostland VE, Ferguson HW (1993) Spironucleus-associated necrotic enteritis in angelfish (Pterophyllum scalare). Can Vet J 34:301-303

Paull GC, Matthews RA (2001) Spironucleus vortens, a possible cause of hole-in-the-head disease in cichlids. Dis Aquat Org 45:197-202

Philbey AW, Andrew PL, Gestier AW, Reece RL, Azey KE (2002) Spironucleosis in Australian king parrots (Alisterus scapularis). Aust Vet J 80:154-160

Poppe TT, Mo TA, Iversen L (1992) Disseminated hexamitosis in sea-caged Atlantic salmon Salmo salar. Dis Aquat Org 14:91-97

Poynton SL, Sterud E (2002) Guidelines for species description of diplomonad flagellates in fish. J Fish Dis 25:15-31

> Poynton SL, Fraser W, Francis-Floyd R, Rutledge P, Reed P, Nerad TA (1995) Spironucleus vortens n. sp. from the fresh-water angelfish Pterophyllum scalare: morphology and culture. J Eukaryot Microbiol 42:731-742

R Development Core Team (2012) R: a language and environment for statistical computing. R Foundation for Statistical Computing, Vienna. Available at www.r-project.org

Rozen S, Skaletsky HJ (2000) Primer3 on the WWW for general users and for biologist programmers. In: Krawetz S, Misener S (eds) Bioinformatics methods and protocols: methods in molecular biology. Humana Press, Totowa, NJ, p 365-386

Sangmaneedet S (1999) Spironucleus vortens of the freshwater angelfish (Pterophyllum scalare): growth requirements, chemotherapeutants, pathogenesis and immunity. PhD thesis, Virginia Polytechnic Institute and State University, Blacksburg, VA

Sangmaneedet S, Smith SA (2000) In vitro studies on optimal requirements for the growth of Spironucleus vortens, an intestinal parasite of the freshwater angelfish. Dis Aquat Org 39:135-141

Sterud E (1998) In vitro cultivation and temperaturedependent growth of two strains of Spironucleus barkhanus (Diplomonadida: Hexamitidae) from Atlantic salmon Salmo salar and grayling Thymallus thymallus. Dis Aquat Org 33:57-61

Editorial responsibility: Sven Klimpel,

Frankfurt, Germany
Sterud E, Poynton SL (2002) Spironucleus vortens (Diplomonadida) in the ide, Leuciscus idus (L.) (Cyprinidae): a warm water hexamitid flagellate found in Northern Europe. J Eukaryot Microbiol 49:137-145

> Tojo JL, Santamarina MT (1998) Oral pharmacological treatments for parasitic diseases of rainbow trout Oncorhynchus mykiss. I. Hexamita salmonis. Dis Aquat Org 33: $51-56$

Uldal A (1996) Life cycle observations on Hexamita salmonis from rainbow trout intestine. In vitro studies. Bull Eur Assoc Fish Pathol 16:112-114

- Uldal A, Buchmann K (1996) Parasite host relations: Hexamita salmonis in rainbow trout Oncorhynchus mykiss. Dis Aquat Org 25:229-231

Whaley J, Francis-Floyd R (1991) A comparison of metronidazole treatments for hexamitiasis in angelfish. Proc Int Assoc Aquat Anim Med 22:108-114

- Williams CF, Lloyd D, Poynton SL, Jørgensen A, Millet COM, Cable J (2011) Spironucleus species: economically important fish pathogens and enigmatic single-celled eukaryotes. J Aquac Res Dev 2 (Spec Iss 2): 002

- Williams CF, Lloyd D, Kolarich D, Alagesan K and others (2012) Disrupted intracellular redox balance of the diplomonad fish parasite Spironucleus vortens by 5-nitroimidazoles and garlic-derived compounds. Vet Parasitol 190:62-73

Woo PTK, Poynton SL (1995) Diplomonadida, Kinetoplastida and Amoebida (Phylum Sarcomastigophora). In: Woo PTK (ed) Fish diseases and disorders, Vol 1. Protozoan and metazoan infections. CAB International, Wallingford, p 27-96

Wood AM, Smith HV (2005) Spironucleosis (Hexamitiasis, Hexamitosis) in the ring-necked pheasant (Phasianus colchicus): detection of cysts and description of Spironucleus meleagridis in stained smears. Avian Dis 49: 138-143

Woolhouse MEJ, Dye C, Etard JF, Smith T and others (1997) Heterogeneities in the transmission of infectious agents: implications for the design of control programs. Proc Natl Acad Sci USA 94:338-342

Submitted: March 11, 2013; Accepted: May 6, 2013

Proofs received from author(s): August 27, 2013 